\title{
Outcome Measures for Gout Clinical Trials: a Summary of Progress
}

\author{
Hugh de Lautour, $M B C h B^{1, *}$ \\ Nicola Dalbeth, FRACP ${ }^{1,2}$ \\ William J. Taylor, PhD, $M B C h B^{3}$
}

\author{
Address \\ ${ }^{*}, 1$ Department of Rheumatology, Auckland District Health Board, 2 Park Road \\ Grafton, Auckland, New Zealand \\ Email: hughdl@adhb.govt.nz \\ ${ }^{2}$ Department of Medicine, University of Auckland, 85 Park Road Grafton, Auckland, \\ New Zealand \\ Email: n.dalbeth@auckland.ac.nz \\ ${ }^{3}$ Department of Medicine, University of Otago, Wellington, 23 Mein Street, \\ Newtown, Wellington, New Zealand \\ Email: will.taylor@otago.ac.nz
}

Published online: 5 March 2015

(C) Springer International Publishing AG 2015

This article is part of the Topical Collection on Gout

Keywords Gout - Outcome · Measurement - Remission - Response criteria

\section{Opinion statement}

Gout is the most common form of inflammatory arthritis. With the development of new agents for the treatment of gout in the last decade, the lack of validated outcome measures for clinical trials in gout has become apparent. Gout has been a topic of discussion at Outcomes in Rheumatology Clinical Trials (OMERACT) meetings since 2002. This work has included identification of core domains for outcome measures for both acute and chronic gout studies. More recently, OMERACT have endorsed a number of these domains and measurement instruments for these domains. The domains appear to be largely relevant to patients with gout but additional issues have been identified, including the absence of an endorsed disease-specific measure of health-related quality of life. Concurrently, a preliminary definition of flare has been developed and leading from this, early versions of composite measures of disease activity and response criteria. With the ability for excellent serum urate control, remission should be possible and preliminary remission criteria have been proposed. Flare definition, response criteria and remission criteria require further validation. The role of serum urate as a biomarker for chronic gout studies is promising, but further work is required to show that serum urate is a biomarker of relevant clinical outcomes. 


\section{Introduction}

Gout is a chronic disease of monosodium urate (MSU) crystal deposition $[1 \bullet \bullet, 2 \bullet]$. Early disease is characterised by intermittent flares of very painful inflammatory arthritis affecting one or more joints [2•]. Treatment of acute gout flares is aimed at reducing pain and swelling and improving the patient's function. With uncontrolled hyperuricaemia, flares become more frequent and severe, with the eventual development of advanced gout characterised by tophi, joint damage and chronic gouty arthropathy. Long-term treatment for gout includes antiinflammatory management of acute flares, antiinflammatory prophylaxis of acute flares and most importantly lowering of serum urate with urate-lowering therapy (ULT).

With the development of new ULT agents such as febuxostat, pegloticase and lesinurad, and antiinflammatory agents such as IL-1 inhibitors, the lack of validated outcome measures for clinical trials in gout has become apparent $[1 \bullet \bullet, 2 \bullet, 3 \bullet]$. Special interest groups have met at Outcomes Measures in Rheumatology Clinical Trials (OMERACT) since 2002 to identify valid outcome measures for gout studies. To date, OMERACT domains have been devised according to the stage of disease, separated as the 'acute gout domain' and the 'chronic gout domain'. With the increased recognition that gout is a chronic disease of MSU crystal deposition, and the development of newer antiinflammatory medications such as canakinumab that treat both the acute attacks and prevent flares, the previously clear distinction between acute and chronic gout has become less well defined [3•]. Nevertheless, this artificial separation has allowed progress in defining core domains and validation of instruments for measurement of different aspects of disease.

The use of serum urate (SU) as surrogate or biomarker associated with control or cure of the disease is widespread and remains the most common marker for treatment response $[4 \bullet \bullet]$. Although it seems clinically obvious that serum urate is a surrogate for clinically meaningful endpoint, this has not been formally shown $[1 \bullet \bullet$, $4 \bullet \bullet, 5]$. Recent work on the definition of a flare and response criteria using a composite measure shows that work on outcome measures for gout studies is coming together to create a useful set of endpoints for future gout studies [6] [7]. This paper will review current domains and measures of outcome in both acute and chronic gout and briefly discuss progress on response and remission criteria.

\section{The OMERACT process}

OMERACT is an independent initiative of international health professionals and patient partners interested in outcome measures in rheumatology which began in 1992. The special interest groups in gout have developed and validated outcome measures in gout through literature reviews, research and consensus exercises $[8-10,11 \bullet, 12 \bullet \bullet$. These domains and measures are subject to the OMERACT filter 1.0 of truth (Does it measure what is intended? captures issues of face, content and construct validity), discrimination (Does the measure discriminate between situations of interest? captures issues of reliability and sensitivity to change) and feasibility (Can the measure be applied easily, given constraints of time, money and interpretability?) [13].

The OMERACT process identified core domains that can be measured in both acute and chronic gout studies, Fig. 1 [14]. Although identified prior to the development of filter 2.0, these domains are aligned with the domain structure proposed by the OMERACT filter 2.0 [15]. More recently, OMERACT have endorsed a number of these domains and their associated specific instruments of measurement for both acute and chronic gout studies, Table $1[4 \bullet \bullet, 8]$. These instruments have passed the OMERACT filter 1.0 of truth, discrimination and 
feasibility and therefore would be considered essential in further gout trials. Despite this progress, a review of recent gout trials showed that only three out of five recommended acute domains were reported and 2.4 out of seven chronic domains $[1 \bullet \bullet$. Similar findings have been reported by Araujo et al. with their review of gout trials showing significant variation in outcome domains and preference towards non-patient-reported measures [16].

\section{Acute gout outcome measures}

The acute gout flare is characterised by a rapid onset of intense pain and swelling in one or more joints, usually developing within hours and then selfresolving over a period of up to 14 days $[2 \bullet, 6]$. Treatment of acute gout flares is aimed at resolution of the attack, typically with non-steroidal anti-inflammatory drugs (NSAIDs), corticosteroids or colchicine; however, there have been recent trials of IL-1 $\beta$ inhibitors [3•]. At OMERACT 9 in 2008, five core domains for acute gout were endorsed: pain, joint tenderness, joint swelling, patient global assessment and activity limitation [8]. There were a number of discretionary domains identified, which are subject to further investigation: acute phase marker, work disability, joint impairment and joint erythema (Fig. 1). At OMERACT 11 in 2012, presentation of a systematic literature review of existing acute gout studies and patient-level clinical trial data led to OMERACT endorsement of measurement for four out of the five domains: pain, joint ten-

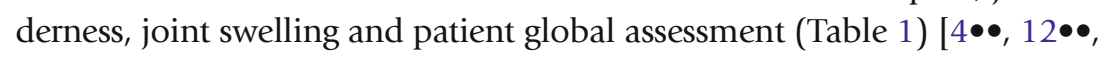
$17 \bullet \bullet$. The wider use of these measures will allow for standardisation of acute gout trial outcomes.

This is the most reported domain in clinical trials with 20 different instruments used to asses pain in available studies with the most common three being $100 \mathrm{~mm}(10 \mathrm{~cm})$ pain visual analogue scale (VAS), 5-point Likert scale and 0-10 numeric rating scale (NRS). These were assessed according to the OMERACT filter. There was a strong correlation of pain with disability as measured by HAQ, but it was consistently weakly correlated with joint swelling. Ceiling and floor effects of pain measures suggested the scale of measurement needed to be wider. Areas for future research of this domain suggested at OMERACT 11 in 2012 included the rate of change of pain, time to pain resolution, minimally and acceptable states of pain and pain at rest versus pain on motion $[4 \bullet \bullet, 12 \bullet \bullet, 17 \bullet \bullet]$. VAS, Likert scale and NRS were all endorsed as measures for pain $[4 \bullet \bullet]$.

This is the second most reported outcome in clinical trials. A 0-3 Likert scale is used in most studies, namely 0 -no joint swelling, 1 -palpable, 2-visible, and 3-bulging beyond the joint margins, in the index joint, as assessed by a physician. Assessment of the index joint in acute gout has high face validity as gout attacks are typically monoarticular. 

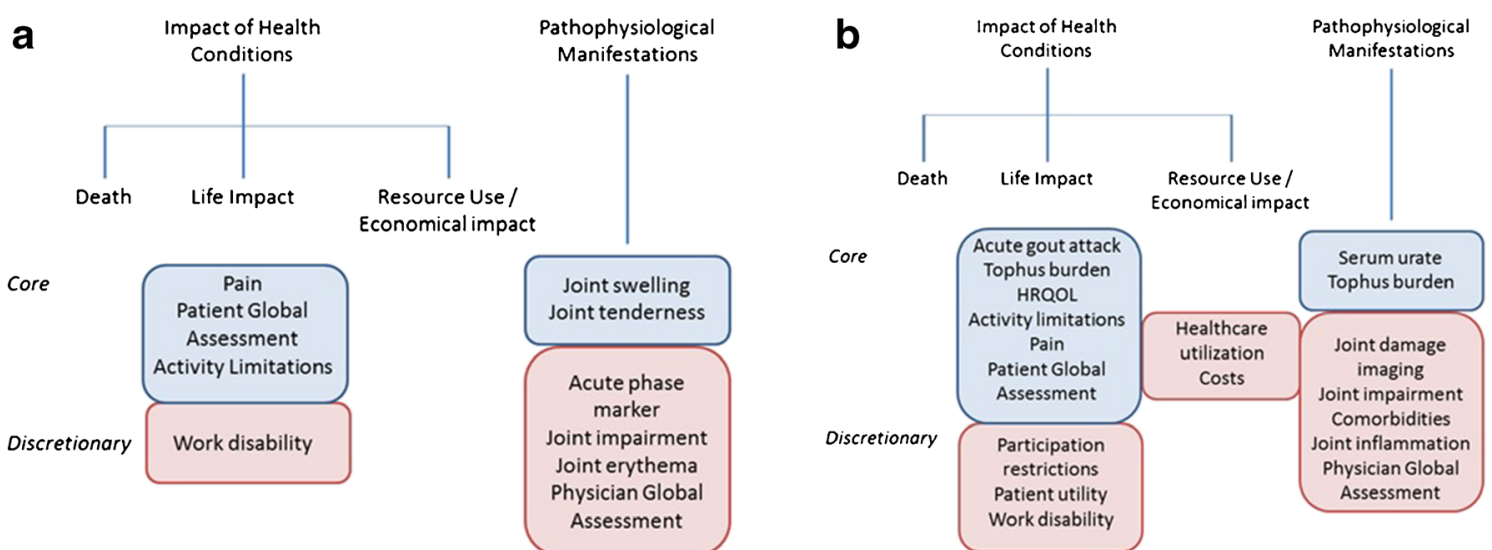

Fig. 1. Acute (a) and chronic (b) domains endorsed by OMERACT for gout studies using the Filter 2.0 framework.

Swollen joint counts and physical measurement of joint circumference have also been used in trials but are limited by inability to assess the degree of swelling in the joint and large variation in joint sizes, respectively $[4 \bullet \bullet, 12 \bullet \bullet, 17 \bullet \bullet]$. A 4 -point physician-completed Likert scale of the index joint was the only measure endorsed by OMERACT for joint swelling $[4 \bullet \bullet]$.

Joint tenderness

This is mostly measured by $0-3$ physician-completed Likert scale of the index joint in trials such as 0 -no pain, 1 -patient states there is pain, 2 -patient states there is pain and winces, and 3-patient states there is pain, winces and withdraws. This had a strong correlation with patient

\section{Table 1. OMERACT endorsed acute and chronic gout domains with associated measurement instruments}

\begin{tabular}{lll}
\hline Context & Endorsed core domains & Endorsed instruments \\
Acute gout studies & Pain & 10-cm VAS, numerical rating scale, Likert type \\
& Joint swelling & 4-point Likert \\
& Joint tenderness & 4-point Likert \\
& Patient global assessment & Global response to treatment by Likert-type scale \\
& Activity limitations & None endorsed \\
Chronic gout studies & Serum urate & Routine biochemical lab testing \\
& Gout flares & None endorsed \\
& Tophus burden & Vernier calliper measurement of long axis (cm) of a sentinel tophus \\
& Health-related quality of life & Short form 36 \\
& Activity limitations & HAQ \\
& Pain & $10 \mathrm{~cm}$ VAS, SF-36 pain subscale \\
& Patient global assessment & $10-\mathrm{cm}$ VAS
\end{tabular}


global assessment and moderate correlation with joint swelling and was endorsed by OMERACT $[4 \bullet \bullet, 12 \bullet \bullet, 17 \bullet \bullet]$.

Patient global assessment

PGA was assessed in most studies with a 0-4-point Likert scale with regard to response to treatment (PGART), for example 'How would you rate the study medication you received for gout? Response options; 0-excellent, 1 -very good, 2-good, 3 - fair, 4- poor' $[4 \bullet \bullet$, $12 \bullet \bullet, 17 \bullet \bullet$. It is important to note the PGA of response to treat-

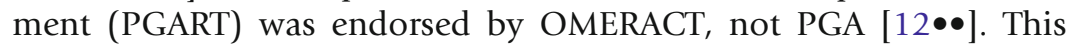
may seem appropriate in acute gout since resolution of symptoms is the goal of therapy. However, there may be issues with this as gout is a highly dynamic disease and rapid changes in clinical status are possible $[12 \bullet \bullet]$. There are issues with recall bias in this measure and results should be interpreted with caution with limited group change

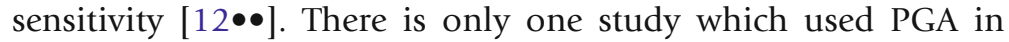
relation to current disease status, and this measure did show within group change sensitivity but due to lack of additional studies has not been endorsed $[12 \bullet \bullet, 17 \bullet \bullet]$.

This has been measured in only a few of studies. Measurement with both the Health Assessment Questionnaire (HAQ) and Medical Outcome Study Short Form-36 (SF-36) physical function subscale (PF) has been reported. Both instruments have been endorsed for chronic gout studies, with HAQ for activity limitation [10]. But no measure of activity limitation for acute gout was endorsed at OMERACT 11 due to lack of evidence for betweengroup differences, noting that only one trial was available for this assessment. It is suggested that future trials continue to include HAQ or HAQ plus an alternative measure so that additional data can be gathered to assess

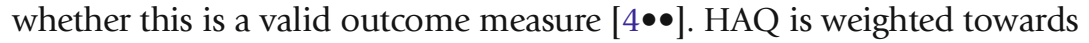
upper limb function, which is not ideal given that gout is typically a lower limb disorder, highlighting a potential need for the development of a new instrument.

\section{Chronic gout outcome measures}

Work though OMERACT has identified nine domains for chronic gout studies: serum urate, flares, tophus burden, health-related quality of life (HRQOL), functional disability, pain, patient global, work disability and joint inflammation. A number of discretionary domains have also been identified, Fig. 1 [14, 18]. At OMERACT 9 (2008) and OMERACT 10 (2010), several measures of chronic gout domains were presented and endorsed: pain with VAS, activity limitation with HAQ, health-related quality of life with SF-36, patient global

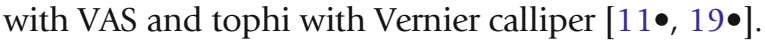


One of the critical outcomes of chronic gout studies is flares $[1 \bullet \bullet, 6,18]$. Before validating gout flares as an outcome, a standardised definition of a flare was required. A preliminary definition of a flare in a patient with established gout has been developed by Gaffo et al. (funded by American College of Rheumatology (ACR)/European League Against Rheumatism (EULAR)) $[6,20]$. In this study, the presence of any patient-reported warm joint, swollen joint, pain at rest score $>3$ or patient-reported flare was independently associated with the gold standard of physician diagnosis of a flare [6]. Several definitions for a flare were proposed including three of the four items, all four items required and a classification and regression tree approach that led to a combination of pain at rest score $>3$ and the presence of gout flare according to the patient [6]. These definitions are purely patient reported which make them feasible for clinical trials. Additionally, adding serum markers of inflammation lead to only minor improvements in the accuracy of flare definition and hence to not justify inclusion to the criteria [6]. To date, none of these definitions have been validated in an external dataset or endorsed by OMERACT, ACR or EULAR, nor is there consensus on how to measure flares as an outcome, number over a time period 'flare burden' or the absence of flares if the treatment is deemed successful $[1 \bullet \bullet]$.

Patient-reported outcomes, pain, patient global assessment, HQQOL and activity limitation

Patient-reported outcomes (PRO) are important in patients with chronic musculoskeletal disorders as they assess the impact of disease and therapy [11 $\bullet$. Patient involvement in OMERACT has only been briefly described, and recent work suggests that concerns of patients with gout are incompletely captured by OMERACT-endorsed domains for chronic gout studies $[1 \bullet \bullet, 21,22]$. Diaz-Torne et al. showed that four areas of importance for patients with gout were represented well by OMERACT core domains: pain, loss of joint motion, work loss and joint swelling and inflammation [22]. However, there were three additional areas ranked highly by patients which were not clearly included within the OMERACT core domains: difficulty with wearing shoes, restrictive diet and sleep [22]. There is, to date, no widely endorsed disease-specific measure of health-related quality of life and further development of a disease-specific measure is required.

During OMERACT 9 in 2008, pain, patient global, HRQOL and activity limitation as domains for outcomes in chronic gout studies were endorsed [11•]. At OMERACT 10 in 2010, validation data was presented for measures in each domain endorsed: pain measurement by VAS, patient global assessment by VAS and activity limitation by Health Assessment Questionnaire Disability Index (HAQ-DI) [11•]. Activity limitation is an important outcome for chronic gout studies as advanced gout is associated with joint destruction, deformity and pain. HAQ-DI is the key PRO in the majority of rheumatology clinical trials and is included in the ACR clinical response criteria for rheumatoid arthritis (RA) [18]. Rasch analysis has shown that HAQ-DI possesses adequate internal validity and clinical 
indices correlated highly with HAQ-DI indicating adequate external validity, in particular with other measures of physical function: SF-36, ACR functional class, Sollerman hand function test, Disability of Shoulder, Arm and Hand Score [18].

A disease-specific measure of HRQOL has been developed for gout, the Gout Assessment Questionnaire (GAQ) and the subsequent GAQ v2.0 $[1 \bullet \bullet, 23]$. Both GAQ and its subset Gout Impact Scale (GIS) which includes gout concern overall, gout medication side effects, unmet gout treatment need, well-being during attack and gout concern during attack have not been endorsed by OMERACT $[11 \bullet, 24]$. This is probably due to concerns of unfamiliarity with the questionnaire, uncertainty on what it measures and issues with internal consistency with questionable psychometric properties $[1 \bullet \bullet, 11 \bullet]$. It has been suggested that an appropriate approach for future the development of a HRQOL instrument would be within the framework of the World Health Organization International Classification of Functioning, Health and Disability (ICF) $[1 \bullet \bullet, 11 \bullet]$. Additionally including further assessment of intermittent versus persistent pain and including other PROs such as sleep, fatigue, social/work participation, diet and measures to encompass all relevant aspects of gout are required.

OMERACT has previous identified serum urate (SU) as an important outcome measure in chronic gout studies, which is understandable given that the underlying biochemical abnormality in gout is hyperuricaemia [5, 14]. Despite all guidelines recommending a treat to a SU target, there is a remarkably small literature that clearly shows that changes in SU in response to treatment are associated with clinically relevant outcomes in randomised controlled trials, with only a trial of pegloticase correlating changes in SU with health-related quality of life and an observational study that showed velocity of tophus regression was associated with degree of urate lowering $[1 \bullet \bullet, 25]$. OMERACT-specific criteria for soluble biomarkers have been developed and adapted for chronic gout studies [5]. At OMERACT 10 in 2010, SU was found to fulfil many of the OMERACT requirements but there was no consensus that it should be endorsed as a biomarker in chronic gout studies, given the lack of research that clearly documents the link between treatment-induced urate lowering and patientrelevant outcomes [5].

Tophus formation is a cardinal feature of advanced gout and is recognised as a domain for chronic gout studies by OMERACT. Tophi have been implicated in the pathogenesis of joint damage and strongly correlate with disability [19•]. Hence regression of tophi is an important outcome. There are eight methods of measurement described to date which range from simple counting of the total number of subcutaneous tophi to imaging with ultrasound (US), magnetic resonance imaging (MRI), CT and dual energy CT for measurement of tophus volume [19•, 26]. The more complex measurements allow analysis of periarticular and tendinous tophi versus 
just subcutaneous tophi and therefore may have greater validity and sensitivity, but this is weighted against additional cost, time and complexity [19•]. At OMERACT, Vernier calliper measurement of subcutaneous tophus diameter was endorsed [19•]. This method however is yet to be used in clinical trials $[19 \bullet, 26]$.

These measures are purely physical and do not quantify the impact of tophi for patients. There was consensus at OMERACT 10 that PRO of tophus burden or impact is important and should be developed. Aati et al. described the development of the Tophus Impact Questionnaire (TIQ-20) which included item generation using qualitative interviews of people with gout and cognitive testing of each item [27]. They generated a preliminary 20-item questionnaire which included items relating to pain, activity limitation, footwear modification, participation, psychological impact and healthcare use due to tophi [27]. Their analysis to date shows the TIQ-20 fulfils the truth and feasibility of the OMERACT filter, but further analysis is required to see whether TIQ-20 scores change in response to treatment [27]. It is possible that the TIQ-20 may complement the GAQv2.0, which is heavily weighted towards the impact of flares to help develop a specific disease measure of HRQOL [27].

\section{Imaging}

Although imaging is not in the inner circle of core domains for chronic gout studies, it is an important outcome measure as joint destruction and tophi are seen in longstanding disease and correlate with HRQOL [24]. To date, the validated imaging measure is plain radiographs with work by Dalbeth et al. showing that a combination of the Sharp-van der Heijde (S-vdH) erosion score and narrowing score most strongly correlated with global consensus scores of S-vdH erosion, S$\mathrm{vdH}$ narrowing, Ratingen destruction and Steinbrocker global scores for assessment of radiological damage in patients with gout [18]. However, with the development of newer imaging modalities, this may change as they have the ability to detect erosive disease and tophi much earlier than plain films [28•].

Both US and MRI have been assessed. US detects around $80 \%$ of tophi seen by MRI with intercondylar, infrapatellar and synovial locations not accessible by US [18]. However, all periarticular tophi detected by MRI are also seen on US, thus making US useful for targeted tophi follow-up [18]. Inter and intraobserver accuracy is satisfactory with the smallest detectable difference of 9 and $4 \mathrm{~mm}$, respectively, and intraclass correlation $>0.8$ (very good) [18]. Given the issues of access and cost of MRI, which limit its feasibility, US may be preferred [9]. Tophus measurement using US has been used in an openlabel ULT trial and has met many of the OMERACT filter requirements [18]. Dual energy CT (DECT) in the future may become the ultimate

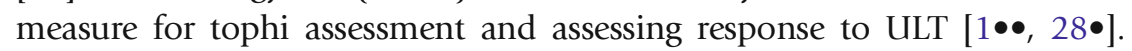
DECT has the ability to evaluate the whole body urate pool, which would be very useful as serum urate imperfectly mirrors the total burden $[1 \bullet \bullet]$. Further validation of DECT is required in the future. 


\section{Response criteria/remission criteria}

With the development of definition of a flare and a core set of outcome measures, the development of response or remission criteria is possible. Composite measures have been used in a number of other rheumatic diseases: rheumatoid arthritis, psoriatic arthritis and ankylosing spondylitis, and work through OMERACT has been done using discrete choice experiments (implemented in 1000Minds) using domains of serum urate, flare frequency, number of tophi and HAQ-DI [29]. There was no unanimous support for this approach but sufficient interest to continue work. Further work by Taylor et al. has cast some doubt on the validity of composite outcomes in gout by showing patients who did not give equal weighting to each component when using a patientbased discrete choice experiment that included domains of flares, pain, serum urate, tophi and activity limitation [21].

On the other hand, preliminary response criteria which are physician based have been presented [7]. The Gout Activity Score (GAS) has been developed using OMERACT core domains and Delphi exercises [7]. Linear regression analysis lead to the development of a composite measure including seven indicators: swollen and tender joint counts, serum urate, tophus size, pain, patient global assessment and number of flares in the last 3 months [7]. GAS was significantly associated with the risk of flares in the next 6 months [7]. Using this measure, it should be possible to define thresholds of low and high disease activity, similar to the Disease Activity Score (DAS) in rheumatoid, and it will be necessary to demonstrate between-group sensitivity to change $[1 \bullet \bullet, 7]$.

Beyond response, given the excellent medication available for gout, remission should be achievable. Initial consensus-building work has been done on this using Delphi methodology and discrete choice experiments [30]. It would appear that a relatively simple definition is possible incorporating domains of serum urate, flares, tophus, pain and PGA with the timeframe for assessment of remission being 12 months [30]. This work is preliminary and will require validation with data sets.

\section{Conclusion}

Work through OMERACT has identified core domains for acute and chronic gout studies and several instruments to measure these. These measures are recommended for all further clinical trials in gout. There is now a preliminary definition of flare and development of response and remission criteria, which will all require further work for validation. Thus, significant progress has been made. Imaging was only briefly mentioned in this paper, but the development of dual energy CT is exciting and may provide a means of assessing the total body urate pool that may help the assessment of biochemical remission. However, there remain several unmet needs in gout outcomes, namely a need for a specific disease measure of HRQOL, further patient participation and validation of serum urate as a biomarker. 


\section{Conflict of Interest}

Hugh de Lautour and William J. Taylor declare that they have no conflict of interest.

Nicola Dalbeth reports personal fees from Takeda, personal fees from Menorini, personal fees from Teijin, personal fees from Savient, grants and personal fees from Ardea, personal fees from AstraZeneca, personal fees from Metabolex, personal fees from Pfizer and grants and personal fees from Fonterra, outside the submitted work.

\section{Human and Animal Rights and Informed Consent}

This article does not contain any studies with human or animal subjects performed by any of the authors.

\section{References and Recommended Reading}

Papers of particular interest, published recently, have been highlighted as:

- Of importance

- Of major importance

1.• Taylor WJ. Measurement of outcome in gout. Indian J Rheumatol. 2013;8:S11-5.

Recent review of gout outcome measures and OMERACT work and need for further development of PROs and disease specific measures.

2.• Rees F, Hul M, Doherty M. Optimizing current treatment of gout. Nat Rev Rheumatol. 2014;10:271-83.

Review of current management of gout including newer ULT.

3. $\quad$ Cavagna L, Taylor W. The emerging role of biotechnological drugs in the treatment of gout. BioMed Res Int. 2014; Article ID 264859. doi:10.1155/2014/264859.

Summary of newer agents emerging for the treatment of gout.

$4 \cdot$ Singh JA, Taylor WJ, Dalbeth N, et al. OMERACT endorsement of measures of outcome for studies of acute gout. J Rheum. 2014;41(3):569-73.

Paper summarising the measures endorsed for acute gout studies at OMERACT 11.

5. Stamp LK, Khanna PP, Dalbeth N, et al. Serum urate in chronic gout - will it be the first validated soluble biomarker in rheumatology. J Rheum.

2010;38(7):1462-6.

6. Gaffo AL, Schumacher HR, Saag KG, et al. Developing a provisional definition of flare in patients with established gout. Arthritis Rheum.

2012;64(5):1508-17.

7. Scire CA, Viroli C, Manara M, et al. Development and preliminary validation of a candidate disease activity score for gout. Ann Rheum Dis. 2013;72(Suppl):48.

8. Schumacher RH, Taylor W, Edwards L, et al. Outcome domains for studies of acute and chronic gout. J Rheum. 2009;36(10):2342-5.
9. Grainger R, Taylor W. Establishing outcome domains for evaluating treatment of acute and chronic gout. Curr Opin Rheumatol. 2008;20:173-8.

10. Grainger R, Taylor W, Dalbeth, et al. Progress in measurement instruments for acute and chronic gout studies. J Rheum. 2009;36(10):2346-55.

11. Singh JA, Taylor WJ, Simon LS, et al. Patient-report outcome in chronic gout: a report from OMERACT 10. J Rheum. 2011;38(7):1452-7.

Presents the PROs assessed and those endorsed at OMERACT 10.

12.• Taylor WJ, Redden D, Dalbeth N, et al. Application of the OMERACT filter to measures of core outcome domains in recent clinical studies of acute gout. J Rheum. 2014;41(3):574-80.

This paper accompanies Ref 4 and Ref 17. It reviews which measures of acute gout domains fulfil the OMERACT filter.

13. Kirwan JR, Boers M, Tugwell P. Updating the OMERACT filter at OMERACT 11. J Rheum. 2014;41(5):975-7.

14. Taylor WJ, Schumacher HR, Baraf HSB, et al. A modified Delphi exercise to determine the extent of consensus with OMERACT outcome domains for studies of acute and chronic gout. Ann Rheum Dis. 2008;67:888-91.

15. Boers M, Kirwan JR, Wells G, Beaton F, Gossec L, d'Agostino, et al. Developing core outcome measurement sets for clinical trials: OMERACT filter 2.0. J Clin Epidemiol. 2014;67(7):745-53.

16. Araujo F, Cordeiro I, Ramiro S, et al. Outcome assessed in trials of gout and accordance with OMERACTproposed domains: a systematic literature review. Rheumatol. 2014;126:1349-19. 
17.• Dalbeth N, Zhong CS, Grainger R, Khanna D, et al. Outcome measures in acute gout: a systematic literature review. J Rheum. 2014;41(3):558-68.

Reviews outcomes meauses used in acute gout studies. Accompanies Ref 4 and Ref 12 .

18. Schumacher HR, Taylor W, Joesph-Ridge N, et al. Outcome evaluations in gout. J Rheum. 2007;34(6):13811385.

19. Dalbeth N, McQueen FM, Singh JA, et al. Tophus measurement as an outcome measure for clinical trials of chronic gout: progress and research priorities. J Rheum. 2011;38(7):1458-61.

A summary of tophus measurements, including imaging.

20. Grainger R, Harrision A, Taylor W. Preliminary identification of potential items for a definition of "gout flare" using Delphi methodology. Ann Rheum. 2005;52(Suppl 9):S105.

21. Taylor W, Brown M, Aati O, Weatherall M, Dalbeth $\mathrm{N}$. Do patient preferences for core outcome domains for chronic gout studies support the validity of composite response criteria? Arthritis Care Res. 2013;65(6):1259-64.

22. Diaz-Torne C, Pou MA, Castellvi I, Corominas H, Taylor WJ. Concerns of patients with gout are incompletely captured by OMERACT-endorsed domains of measurement for chronic gout studies. J Clin Rheumol. 2014;20(3):138-40.

23. Colwell HH, Hunt BJ, Pasta DJ, Palo WA, Mathias SD, et al. Gout Assessment Questionnaire: initial results of reliability, validity and responsiveness. Int J Clin Pract. 2006;60(1):1210-7.
24. Terkeltaub R, Khanna D, Singh J, Sarkin A, Hirsch JD, et al. Gout disease-specific quality of life and the association with gout characteristics. Patient Relat Outcome Measures. 2010;1:1-8.

25. Perez-Ruiz F, Calabozo M, Pijoan JI, HerreroBeites AM, Ruibal A. Effect of urate-lowering therapy on the velocity of size reduction of tophi in chronic gout. Arthritis Rheum. 2002;47:35660.

26. Dalbeth N, Schauer C, MacDonald P, et al. Methods of tophus assessment in clinical trials of chronic gout: a systemic literature review and pictorial reference guide. Ann Rheum Dis. 2011;70:597-604.

27. Aati O, Taylor WJ, Siegert RJ, et al. Development of a patient-reported outcome measure of tophus burden: the Tophus Impact Questionnaire (TIQ-20). Ann Rheum Dis. 2014. doi:10.1136/annrheumdis-2014205671.

28. McQueen F, Reeves Q, Dalbeth N. New insights into an old disease: advanced imaging. Postgrad Med J. 2013;89:87-93.

Good review of imaging modalities in gout including DECT.

29. Taylor W, Singh JA, Saag KG, et al. Bringing it all together: a novel approach to the development of response criteria for chronic gout clinical trials. J Rheum. 2011;38(7):1467-70.

30. de Lautour H, Dalbeth N, \& Taylor W. Development of preliminary remission criteria for gout using Delphi and 1000Minds consensus exercises. Arthritis Rheum. 2014 Suppl (ACR/ARHP Annual Meeting Abstract Supplement) 66(suppl):S68. 